\title{
Numerical Study on Relaminarization in Fish-Like Locomotion
}

\author{
by Zuogang Chen*, Member
}

\author{
Yasuaki Doi*, Member
}

\begin{abstract}
Summary
Unsteady viscous flow field around a fish-like advancing NACA wing is numerically studied. The main objective is to study the abilities of a flexible fish-like body to produce thrust, achieve higher propulsive efficiency as well as to catch the basic flow characteristics in viscous flow field. The Reynolds numbers based on the oncoming velocity and the body length range from $10^{6}$ to $3 \times 10^{6}$. The flow is simulated by solving two-dimensional unsteady Reynolds averaged Navier-Stokes equation in a primitive value formulation, while the turbulence transportation is described by updated algebraic eddy-viscosity model and transitional zone is computed by empirical formula. The numerical scheme is based on the MAC method with a body fitted coordinate system. The simulation shows that the high propulsive efficiency of fish-like locomotion comes from relaminarization and effective vorticity control. Relaminarization reduces the frictional force at higher Reynolds number. Fish-like locomotion can recapture energy contained in the eddies by skillfully controlling vorticity generation, travel and shed through the body undulation.
\end{abstract}

\section{Introduction}

The flow field around a swimming fish or cetacean has been investigated for a long time by the researchers in various field of study such as biology, physical science and engineering. This interest has been inspired not only to understand and simulate an efficient swimming propulsion, but also to utilize the results for engineering application. Fish may propel themselves by successively generating and destroying tail tip eddies of alternating direction, which is believed as the main reason for high efficiency of fast-starting and maneuvering. A foil moving forward at steady speed and oscillating harmonically in a combination of lateral motion (heave) and angular motion (pitch) may produce thrust under proper parametric conditions ${ }^{1)}$. It is thought that the tail of a fish controls vortices generated by the main body of fish with the intent of recovering some of the energy lost in forming these vortices ${ }^{2)}$. Also, an oscillating foil can be used to alter and reposition oncoming vorticity ${ }^{3)}$ and recapture energy contained in the eddies of an oncoming flow using vorticity control ${ }^{1,4)}$. Two

* Graduate School of Engineering, Hiroshima University dimensional flow studies show that the downstream flow may be characterized by the formation of a sinuous wake; or a wake that has either two large or four vortices per period. High propulsive efficiency is associated with the formation of two vortices per cycle, forming a staggered array of vortices resembling a Karman vortex street behind bluff bodies, but with the vortices rotating in opposite directions. The process of repositioning the oncoming vorticity is a crucial part of the interaction. As identified in Gropalkrishnan et al. ${ }^{1)}$ and Streitlien et al. ${ }^{4}$, a fish may employ a vorticity control scheme similar to a definitive mode to recover some of the energy lost in forming these vortices.

Another reason for high propulsive efficiency is so-called relaminarization. Taneda and Tomonari ${ }^{5)}$ found that turbulence in the boundary layer of an undulating plate was suppressed when the speed of propagation of the undulating wave exceeded the free stream velocity. Chen and Doi ${ }^{\text {9) }}$ numerically showed that the increase of wave propagation velocity reduced the frictional force. Wolfgang et al. $^{7}$, Anderson et al. ${ }^{8)}$ and Techet et al. ${ }^{9)}$ obtained experimental data of velocity profiles in boundary layer of a swimming fish. They thought that undulating motion converted turbulent flow into laminar flow at some domain, 
which was called as relaminarization. Experiments ${ }^{10)}$. 11) demonstrated the ability of flexible-hull vehicle to achieve high maneuverability and turbulence reduction through flow control. Even Barrett et al. ${ }^{(1)}$ pointed out that the power required to propel an actively swimming, streamlined, fish-like body was significantly smaller than the power needed to tow the body straight and rigid at the same speed.

Although the possibility of relaminarization has been found by experiment, direct flow measurements on live fish has a limitation of disclosing the more detailed mechanism. Numerical computation supports to study the mechanism more precisely. In the present paper, relaminarization in boundary layer of swimming body is disclosed by numerical simulation. The flow information near the swimming fishlike body is shown and the drag reduction mechanism is tried to be explained. Vorticity generation, travel and shed are studied as a whole. At present the simulation is limited to the two-dimensional flow, which is thought as an essential step for further development.

\section{Modeling of undulation}

The simulated undulating body is NACA series wing, which undulates actively in unbounded oncoming flow, where $x$ and $y$ are the longitudinal and the lateral coordinates whose origin locates at the leading edge of the body. All variables are normalized by the body length $L$ and oncoming velocity $U$, as well as the time is normalized by $L / U$. The movement of the undulating body camber line in $y$ direction is given as following,

$$
y_{r}(x, t)=a x^{\prime \prime} \sin [2 \pi b(x-S p \cdot t)]
$$

where $a$ is amplitude, $n=1.1,2 \pi b$ is wave number, $S p$ is phase velocity, $t$ is time, $0 \leqq x \leqq X e n d(t)$ where $\operatorname{Xend}(t)$ is $x$-coordinate of the trailing edge calculated as follows,

$$
\int_{0}^{\operatorname{Xend}(t)} \sqrt{1+\left(\frac{\partial y_{c}}{\partial x}\right)^{2}} d x=1.0
$$

Eq.(2) keeps the body length constant during the undulation. The body surface is given by keeping the deformed thickness line length constant. The positive phase velocity propagates waves from the leading edge toward the trailing edge. Typical loci during one period can be seen in Fig. 1 at chosen parameters: $a=0.051, b=1.0 . T$ is undulating period while $0 / 8 T$ corresponds to the time when the tailing edge reaches its maximum lateral position and the others denote the configurations after every $1 / 8$ period. The Reynolds numbers based on the oncoming velocity $U$ and body length $L$ range from $10^{6}$ to $3 \times 10^{6}$.

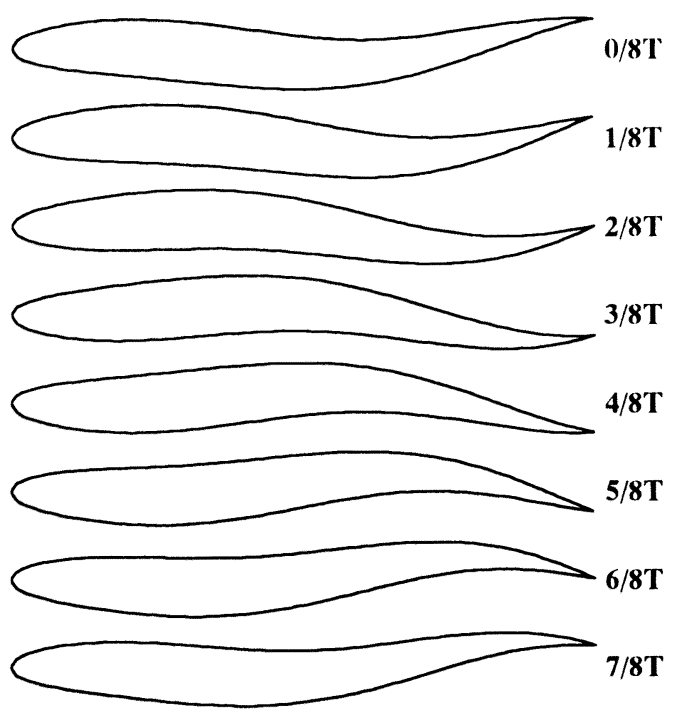

Fig.1 Configurations of an undulating NACA0010 during one period

\section{Numerical scheme}

The governing equations are Reynolds averaged Navier-Stokes equation (3) and continuity equation (4)

$$
\begin{aligned}
& u_{t}+u u_{x}+v u_{y}=-p_{x}+\frac{1}{\operatorname{Re}} \nabla^{2} u+R_{x} \\
& v_{t}+u v_{x}+v v_{y}=-p_{y}+\frac{1}{\operatorname{Re}} \nabla^{2} v+R_{y} \\
& u_{x}+v_{y}=0 \\
& R_{x}=\left\{v_{t} \cdot\left(2 u_{x}\right)\right\}_{x}+\left\{v_{t}\left(u_{y}+v_{x}\right)\right\}_{y} \\
& R_{y}=\left\{v_{t}\left(v_{x}+u_{y}\right)\right\}_{x}+\left\{v_{t} \cdot 2\left(v_{y}\right)\right\}_{y}
\end{aligned}
$$

Subscripts represent partial differentiations with respect to the referred variables except eddy viscosity $v_{t}$ and $R_{x}, R_{y}$, which are Reynolds stress components expressed by eq.(5). In eqs.(3), (4) and (5), $u$ and $v$ are $x$ and $y$ components of velocity, $p$ is pressure, $R e$ is Reynolds number. The governing equations are solved in a primitive value formulation. A numerical co-ordinate transformation is introduced into a body fitted curvilinear coordinate system to simplify the computational domain and to facilitate the implementation of boundary conditions. H-type grid system is adopted, but near the leading edge the grid is modified to be adapted for the blunt body head.

The numerical scheme is based on the MAC method where the momentum equation is solved by the time marching method on the body fitted coordinate system. The oncoming velocity and the undulating amplitude increase smoothly from zero at $t=0$ to the unit at $t=t_{0}$ 
( $t_{0}$ : one specified value). After that the oncoming velocity and undulating amplitude keep constant. The first order difference form of the time derivative is used for an explicit advancement in time. The convection terms are discretized by the third order upwind scheme, while all the other spatial derivatives are discretized by the second order central difference scheme. On the body surface, no-slip condition is applied for the velocity. For the pressure, Neumann type condition is applied to satisfy the momentum equation. A uniform pressure is applied on the inflow boundary, while a zero-gradient extrapolation is used on the outlet boundary. The Poisson equation for pressure is solved by using Successive Over Relaxation method.

The computational domain is $-3.0 \leqq x \leqq 10.0,-2.0 \leqq$ $y \leqq 2.0$, where the coordinates are normalized by body length $L$. The grid consists of $[250 \times 73]$ points in $x, y$ directions where minimum normal spacing is $6.06 \times 10^{-5}$ for $R e=2 \times 10^{6}$.

To simulate turbulent flow, Baldwin-Lomax model $^{(2)}$ is applied for eddy viscosity $v_{t}$. As the wake field of the undulating body is asymmetric, the modified Baldwin-Lomax model proposed by Paterson and Stern $^{13)}$ has been adopted. They applied the model for unsteady flow field given by the MIT flapping foil experiment and validated the model as an effective simulation for unsteady flow. Therefore in the present method, the updated model ${ }^{13)}$ is used to calculate eddy viscosity $v_{t}$ in eq.(5).

The transition onsets when the non-dimensional $v_{t}$ is larger than $14^{12}$, while the transitional zone ends where $v_{t}$ is larger than 25 .

\section{Computed results and discussions}

\subsection{Pilot computation}

The validation for laminar simulation has been performed through the comparison of computed velocity profile with analytical solution of a flat plate $^{14)}$. To verify the updated computer code, turbulence flow around a flat plate was numerically simulated at $R e=10^{6}$. Fig. 2 shows the computed $y^{+} v s$ $u^{+}$, where $u^{+}$and $y^{+}$are frictional stress velocity and wall coordinate respectively. The flow are laminar at $x=0.1091$ and transitional at $x=0.5933$. It can be found that the velocity profiles show good agreement with the theoretical solution when the flow has been fully developed at $x=0.8537$ and $x=0.9494$.

The computed drag coefficient for NACA0012 at Reynolds number $2.8 \times 10^{6}$ is $6.7 \times 10^{-3}$, which shows good agreement with the experimental result, $6.6 \times 10^{-3}$ in Abbott ${ }^{15}$. In Fig.3, the computed pressure coefficients also give agreement with experimental $\operatorname{data}^{16)}$

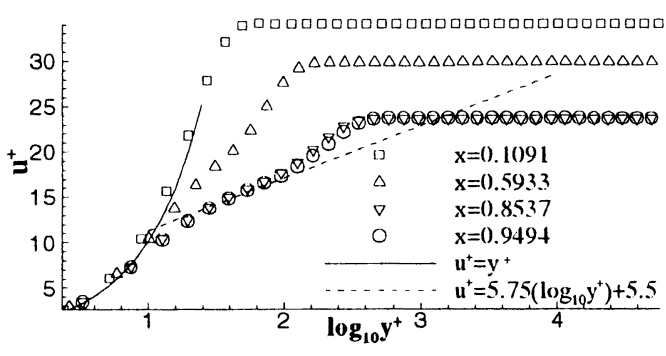

Fig. 2 Computed $y^{+}$vs $u^{+}$for a flat plat at $R e=10^{6}$

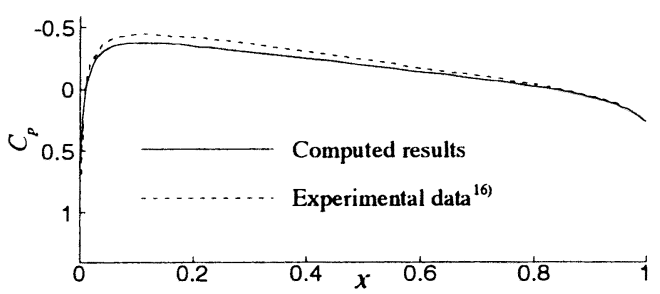

Fig. 3 Surface pressure distribution for NACA0012 at $R e=2.8 \times 10^{6}$

\subsection{Self-propulsion condition}

The total force acting on the body varies during the undulating motion. Therefore, in the present study, self-propulsion is defined as the condition when the time-averaged total force becomes zero. The amplitude $a$ and wave number $2 \pi b$ are chosen firstly and the phase velocity $S p$ is tuned to meet the self-propulsion condition. The related parameters are as follows,

$$
\begin{gathered}
\bar{C}_{F x}=\frac{\frac{\int_{0}^{T} F_{x f} d t}{T}}{\frac{1}{2} \rho U^{2} S} \quad \bar{C}_{P x}=\frac{\frac{\int_{0}^{T} F_{x p} d t}{\frac{1}{2} \rho U^{2} S}}{U \cdot \int_{T} D d t} \\
\bar{C}_{T x}=\bar{C}_{F x}+\bar{C}_{P x} \\
\int_{P} \frac{\int_{T} \text { outputdt/T}}{\int_{T}^{\text {inputdt } / T}}=\frac{\left.\int_{T} \int_{x s}\left(\tau_{x}+p_{x}\right) \cdot u d s+\int_{s}\left(\tau_{y}+p_{y}\right) \cdot v d s\right] d t}{}
\end{gathered}
$$

where $F_{x f}$ is $x$-component of frictional force exerted on the body, $F_{x p}$ is $x$-component of pressure exerted on the body, $T$ is the period of the undulation, $\rho$ is the density of the fluid, $U$ is the oncoming velocity, $S$ is the area of the body with unit width. The negative symbol of pressure component $F_{x p}$ (against to $x$-axis) represents thrust and the positive symbol of frictional force component $F_{x f}$ (same to $x$-axis) represents resistance. When the self-propulsion condition is achieved, the time-averaged total force exerted on the 
body becomes zero. Thus the propulsive efficiency $\eta_{P}$ is defined by eq.(7) in the present study. The output work rate is the product of the oncoming velocity $U$ and the resistance $D$ of a straightly advancing rigid body at the same Reynolds number. The input work rate is the work rate exerted on the body to produce the undulation. In eq.(7), $\tau_{\mathrm{x}}, p_{x}$, $\tau_{\mathrm{y}}$ and $p_{y}$ are $x$ or $y$-component of frictional stress or pressure exerted on the body, $u$ and $v$ are $x$ and $y$-components of the velocity, $\pm S$ indicates the both sides area of the body.

\subsection{Drag reduction and relaminarization}

Turbulent flow around an undulating NACA0010 was carried out. Fig.4 shows the variations of propulsive efficiency and frictional force coefficient. $C_{F O}$ is the frictional force coefficient for rigid body while $\bar{C}_{F x}$ is that for undulating body at self-propulsion state. At low Reynolds number, $\bar{C}_{F x}$ is over $C_{F O}$ by more than $70 \%$, but at enough higher Reynolds number, $\bar{C}_{F x}$ is smaller than $C_{F O}$. Table 1 lists the values of drag reduction and propulsive efficiency for NACA0010 at $a=0.051, b=1.0$. Frictional resistance reduction is about $22 \%$ at $R e=2 \times 10^{6}$ and about $32 \%$ at $R e=3 \times 10^{6}$. It is found that the propulsive efficiency defined by eq.(7) is greater than 1 at $R e=2 \times 10^{6}$ and $R e=3 \times 10^{6}$. It numerically shows that the power required to propel an actively swimming fish-like body is smaller than the power needed to tow the body straight and rigidly at the same speed.

Fig.5 shows the distributions of unsteady local frictional stress coefficients on the upside body surface during one undulating period at $R e=2 \times 10^{6}$. The thicker line plots the frictional stress for a rigid body. In the figure, $x=0$ and $x=1$ represent the leading and trailing edge respectively. As shown in Fig.5, the frictional stress on undulating body surface obviously diminishes on most part of the body $(0.3 \leqq x \leqq 0.9)$. Fig. 6 plots the calculated transition zone. During a period, the laminar flow domain is enlarged by the undulating motion, which implies that the fish-like undulation delays the turbulence transition. Fig.7 compares the velocity profiles at $x=0.1416,0.3510$ and 0.7617 . The thicker lines plot the velocity profiles for a rigid body, while the marks represent the unsteady profiles during one undulating period. The dashed line plots the velocity of logarithm law. It is found, at $x=0.1416$, the undulation makes the profiles deviate laminar form in a small degree, which increases local frictional stress as shown in Fig.5. While at $x=0.3510$, the undulation makes the turbulent flow relaminarize at some time instants. At $3 / 8 T$, $4 / 8 T$ and $5 / 8 T$, the profiles deviate from logarithm law form, so the frictional force is reduced greatly as shown in Fig.5. Meanwhile, the profiles at $x=0.7617$ are close to the logarithm law, but the undulation decreases the magnitude of the frictional stress.

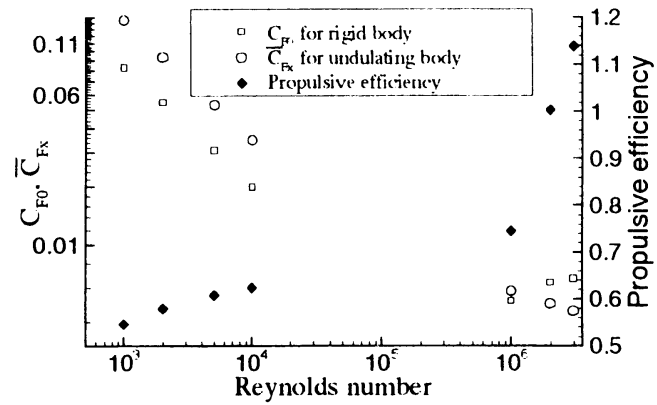

Fig.4 Variations of fictional force coefficient and propulsive efficiency with Reynolds number for NACA0010

Table 1 Drag reduction and propulsive efficiency for NACA0010 at $a=0.051, b=1.0$

\begin{tabular}{|c|c|c|c|c|c|}
\hline$R e$ & $C_{F 0} \times 10^{2}$ & $S p$ & $\bar{C}_{F_{x}} \times 10^{2}$ & $\begin{array}{c}\left(\bar{C}_{F x} /\right. \\
\left.C_{F 0}\right)^{-1}\end{array}$ & $\eta P$ \\
\hline $10^{6}$ & 0.5115 & 1.2313 & 0.5782 & $13.0 \%$ & 0.745 \\
\hline $2 \times 10^{6}$ & 0.6362 & 1.1997 & 0.4948 & $-22.2 \%$ & 1.009 \\
\hline $3 \times 10^{6}$ & 0.6647 & 1.1786 & 0.4529 & $-31.9 \%$ & 1.135 \\
\hline
\end{tabular}

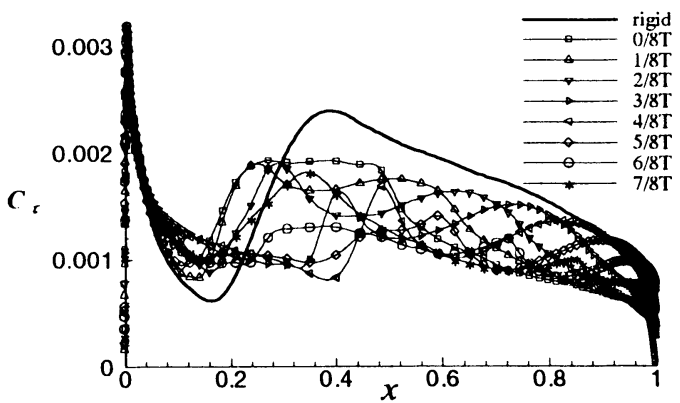

Fig. 5 Distributions of local frictional stress coefficient for NACA0010 at $R e=2 \times 10^{6}$

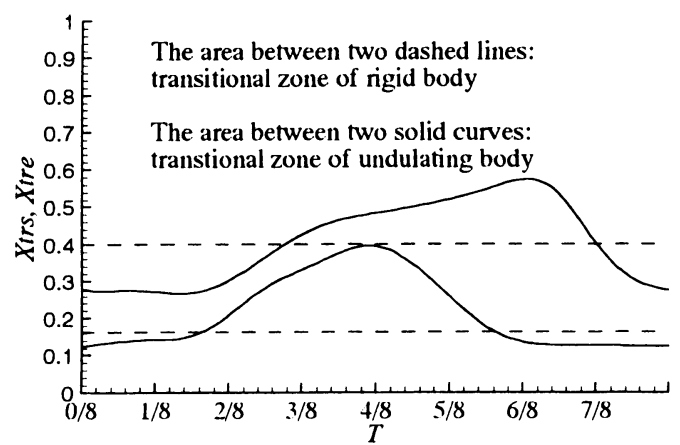

Fig. 6 Transition zone variations caused by undulation at $R e=2 \times 10^{6}$ for $\mathrm{NACA} 0010$ 

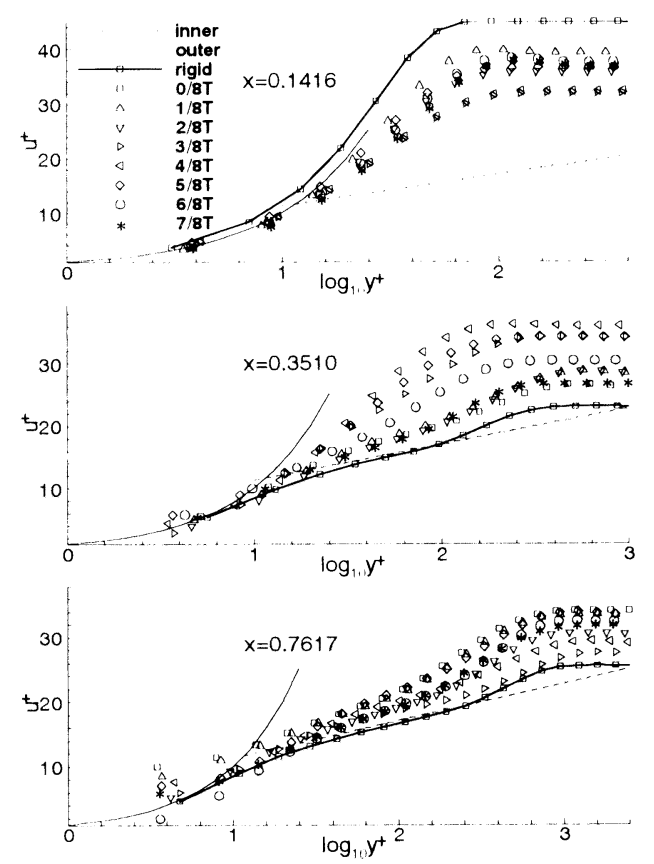

Fig. 7 Effects on velocity profiles by undulation for NACAOO) 10 at $R e=2 \times 10^{6}$

Fig. 8 plots the variations of pressure gradient in $x$-direction at the mesh points most near the upside body surface. The undulation reduces the mean pressure gradient at almost whole zone, which may be the main reason for turbulence suppression. The corresponding flow phenomena can be observed in Figs.9 and 10, which respectively plot the distributions of vorticity $|(\omega)|$ and relative velocity $u_{\text {ref }}$ at the mesh points most near the upside body surface. The relative velocity $u_{r e f}$ denotes $x$-component velocity subtracted by velocity component on the body surface. From Fig.9, globally the vorticity becomes smaller under undulation, which implies that turbulence strength is also reduced because the eddy viscosity is bound to diminish by mixing length formula ${ }^{12}$. From Fig.10, when $x$ ranges between 0.3 and 0.9 , the relative velocities $u_{r e f}$ are smaller than those for rigid case. The decrease of relative velocity leads to the reduction of frictional stress.

Fig. 11 shows the variations of hydraulic coefficients with phase velocity for NACAOO)10 at $R e=2 \times 10^{6}$, $a=0.051, b=1.0$. When $S p=1.2, \bar{C}_{T x}$ becomes zero so self-propulsion is achieved. When $S p$ is larger than 1.2 , the net thrust can be obtained. The present study evaluates the propeller efficiency when the flexible-hull body is taken as a propulsor. The related propeller efficiency $n$ is defined as following,

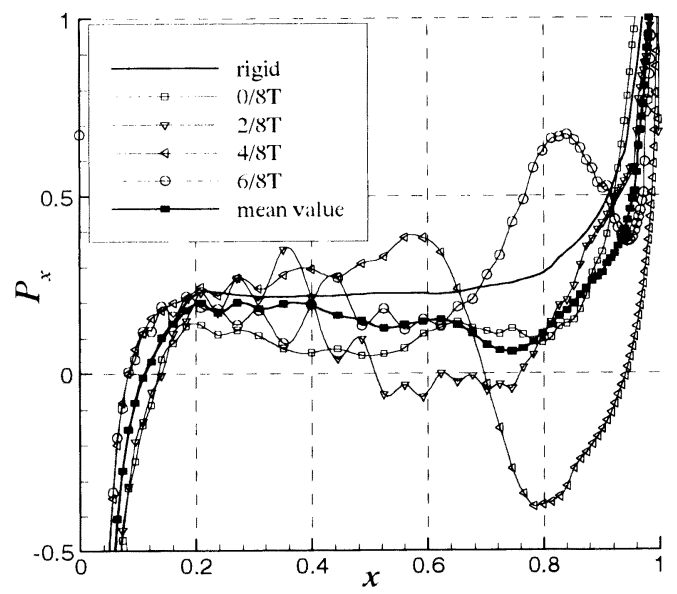

Fig. 8 Pressure gradient in $x$-direction at mesh points most near the upside body surface

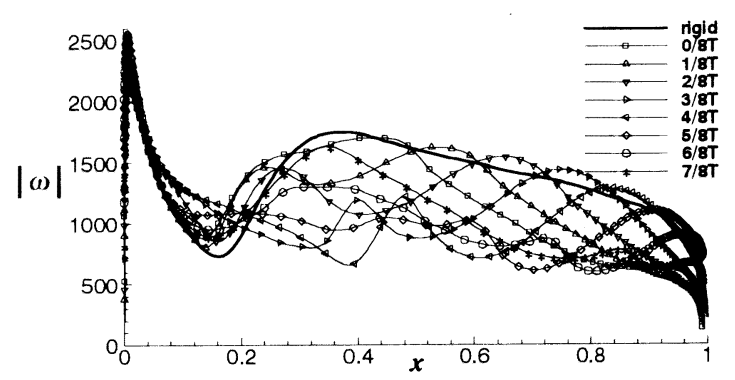

Fig.9 Comparisons of vorticity at mesh points most near the upside body surface

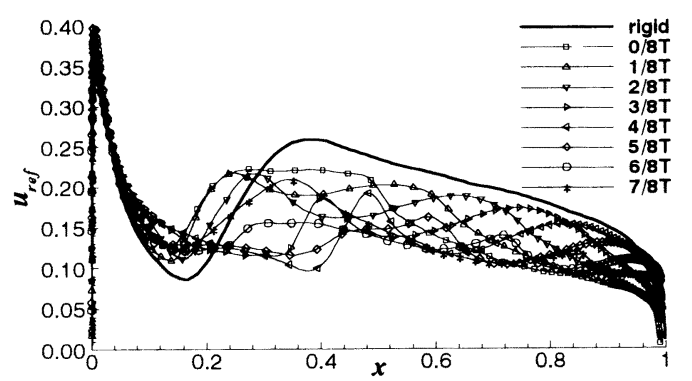

Fig.10 Comparisons of relative velocity at mesh points most near the upside body surface

$\eta=\frac{\int_{T}^{\text {outputdt } / T}}{\int_{T}^{\text {inputdt } / T}}=\frac{U \cdot \int_{T} T_{x} d t}{\int_{T} \int_{ \pm \leq}\left(\tau_{x}+p_{x}\right) \cdot u d s+\int_{ \pm s}\left(\tau_{y}+p_{y}\right) \cdot v d s \mid d t}$

where the input work rate is defined as same as that in eq.(7) for $\eta_{P}$, the output work rate is the product of net thrusting force $T_{x}$ and oncoming velocity $U$. Certainly $\eta$ is zero for self-propulsion state, just like case 1 in the Table 2. Cases 2, 3 and 4 represent the 
computed results for NACA0010 at different phase velocity. With the increase of $S p, \eta$ increases monotonously when $S p$ ranges from 1.2 to 1.6. The difference between case 4 and case 5 is the body thickness. The thinner flexible-hull can achieve higher efficiency and generate larger thrust. The comparison between cases 5 and 6 explores that 11 increases with Reynolds number. The high efficiency of $\eta$ shown in Table 2 indicates that fish-like undulating body can be used as a propeller because of its satisfied propulsive performance at higher Reynolds number.

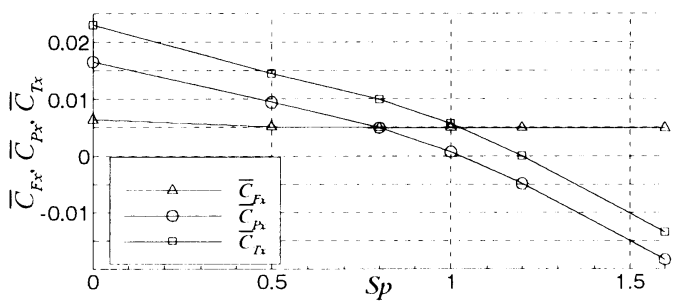

Fig.11 Hydraulic coefficients variations with phase velocity for NACA0010 at $R e=2 \times 10^{6}, a=0.051, b=1.0$

Table 2 Investigation on propeller efficiency

\begin{tabular}{|c|c|c|c|c|c|}
\hline Case & $R e$ & $\begin{array}{c}\text { Relative } \\
\text { thickness }\end{array}$ & $S p$ & $\bar{C}_{T x} \times 10^{2}$ & $\eta$ \\
\hline 1 & $2 \times 10^{6}$ & $10 \%$ & 1.2 & -0.0011 & 0 \\
\hline 2 & $2 \times 10^{6}$ & $10 \%$ & 1.3 & -0.3211 & 0.2992 \\
\hline 3 & $2 \times 10^{6}$ & $10 \%$ & 1.5 & -0.9986 & 0.5019 \\
\hline 4 & $2 \times 10^{6}$ & $10 \%$ & 1.6 & -1.353 & 0.5389 \\
\hline 5 & $2 \times 10^{6}$ & 0 & 1.6 & -1.935 & 0.6264 \\
\hline 6 & $3 \times 10^{6}$ & 0 & 1.6 & -1.981 & 0.6438 \\
\hline
\end{tabular}

\subsection{Streamline pattern study}

The basis for enhancing performance through unsteady flow control is the formation of large-scale vortices through body motion, the sensing and manipulation of these vortices as they move down the body, and the eventual repositioning through tail motion. These concepts constitute the essence of vorticity control. Fig. 12 shows streamlines around NACA0010 at $R e=2 \times 10^{6}, a=0.051, b=1.0, S p=1.2$. The body sheds one vortex per half period when the tail is near its maximum lateral position $(0 / 8 T)$. The vortices shed from the tail and fade in the wake. They resemble reverse Karman vortex street.

The hydraulic coefficients at different phase velocities have been displayed in Fig.11. Some streamlines are shown in Figs.13 and 14, which plot the streamlines when the tail reaches the maximum lateral position for phase velocity $S p$ of 0.0 and 1.6.
When $S p$ equals (0.), no clear vortices exist in the wake. Due to the obstruction of flow by the tail, the pressure on the upside body is obviously larger than the pressure on the downside as shown in Fig. 13. The pressure drag $C_{P, x}$ is very large as shown in ligg. 11. The tail slips in the fluid and forms tortuous streamlines. When $S_{p}$ equals 1.6, due to the propagating wave, there exist shedding vortices in the wake. The propagating undulation improves the pressure distributions exerted on both sides. On the downside near the tail, the existed vortex forms a zone with higher pressure. Correspondingly on the other side there exists a lower pressure zone where streamlines are almost normal to the body surface. The pressure difference near the tail generates larger thrust and forms regular shedding vortices and smooth streamlines in the wake. The above phenomena can be even easily observed in laminar case simulated by Chen and Doi") (Fig.5a and Fig.5b). Thus the presence of vorticity in the wake of an undulating body in viscous fluid is a consequence of the need for a propulsive jet to counter the body drag.
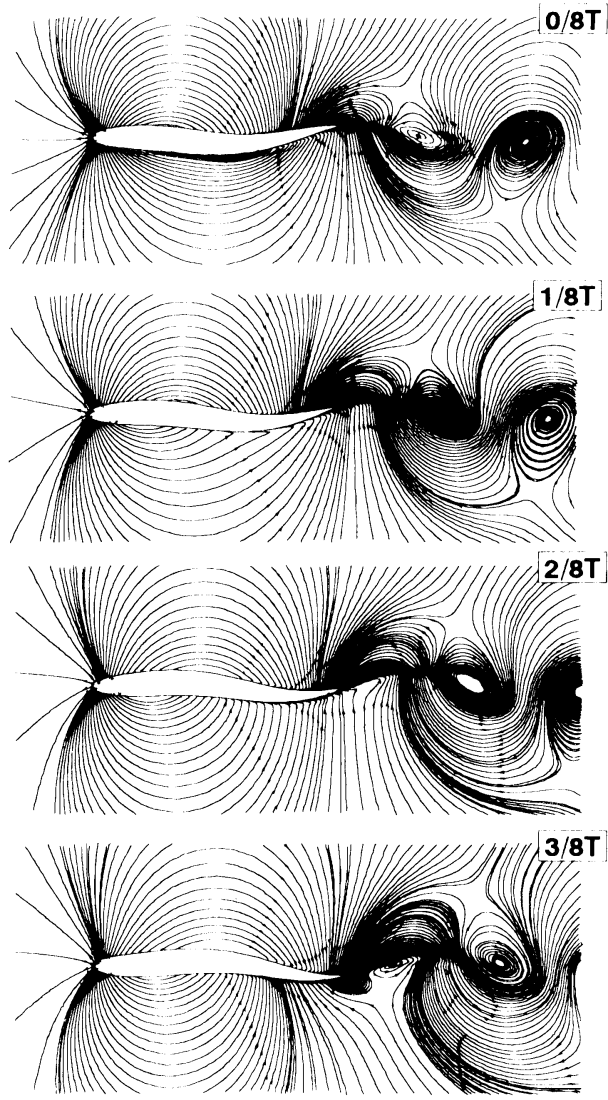

Fig.12 Streamline distributions for NACA0010 at $R e=2 \times 10^{6}, a=0 .(051, b=1.0, S p=1.2$ 


\section{References}
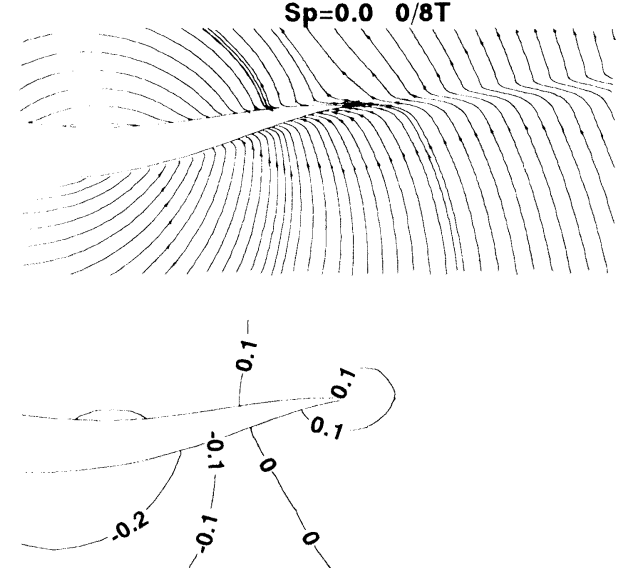

Fig.13 Comparison of streamlines and pressure distribution for NACAOO10 at $R e=2 \times 10^{6}, a=0.051$, $b=1.0, S p=0.0$

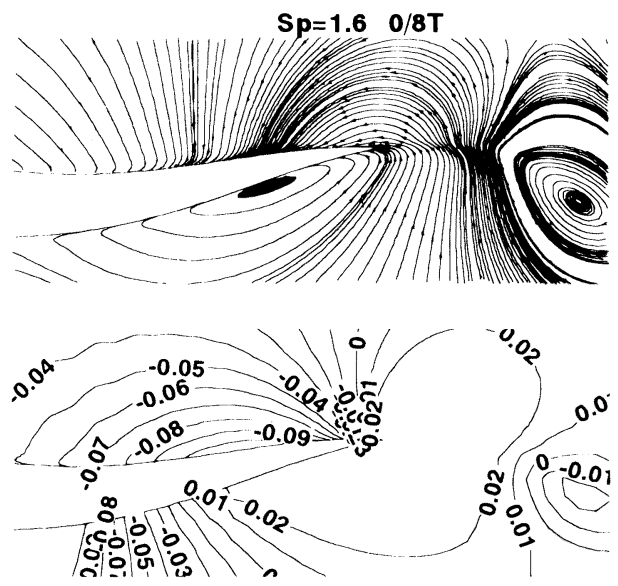

Fig. 14 Comparison of streamlines and pressure distribution for NACAOO 10 at $R e=2 \times 10^{6}, a=0.051$, $b=1.0, S p=1.6$

\section{Conclusions}

The MAC method with a body fitted coordinate system is applied to simulate the turbulent flow around an undulating advancing NACA section. A modified turbulence model is adopted for Reynolds averaged equation. The simulations show that fish-like locomotion can reduce frictional resistance by flow relaminarization and achieve the propulsive efficiency defined by eq.(7) more than 1 . Vorticity travel shows close relation to pressure distribution. Fish-like undulating body shows the potential as a propulsor.
1) Gopalkrishnan, R., Triantafyllou, M. S., Triantafyllou, G. S. and Barrett, D.: "Active Vorticity Control in a Shear Flow Using a Flapping Foil", Journal of Fluid Mechanics, Vol. 274, (1994), pp.1-21.

2) Anderson, J. M., Streitlien, K., Barrett, D. S. and Triantafyllou, M. S.: "Oscillating Foils of High Propulsive Efficiency", Journal of Fluid Mechanics. Vol. 360, (1998), pp.41-72.

3) Cortelezzi, L., Chen, Y.-C. and Chang, H.-L.: "Nonlinear Feedback Control of the Wake past a Plate: From a Low-Order Model to a Higher-Order Model", Physics of Fluids, Vol. 9, No. 7, (1997), pp.2009-2022.

4) Streitlien, K., Triantafyllou, G. S. and Triantafyllou, M. S.: "Efficient Foil Propulsion Through Vortex Control", AIAA Journal, Vol. 34, No. 11, (1996), pp.2315-2319.

5) Taneda, S. and Tomonari, Y.: “An Experiment on the Flow around a Waving Plate, Journal of the Physical Society of Japan", Vol. 36, No. 6, (1974), pp.1683-1689.

6) Chen, Z. and Doi, Y., "Numerical Simulation on Laminar-Turbulent Flow around Fish-Like Advancing Wing", Transactions of The West-Japan Society of Naval Architects, Vol. 103, (2001), pp.245-253.

7) Wolfgang, M. J., Tolkoff, S. W., Techet, A. H., Barrett, D. S., Triantafyllou, M. S., Yue, D. K. P., Hover, F. S., Grosenbaugh, M. A., Mcgillis, W. R., "Drag Reduction and Turbulence Control in Swimming Fish-like Bodies", In Proceeding International Symposium Seawater Drag Reduction, Newport, RI pp.463-469, July, 1998.

8) Anderson, E. J., Mcgillis, W. R. and Grosenbaugh, M. A., "The Boundary Layer of Swimming Fish", The Journal of Experimental Biology, 204, pp.81-102, 2001.

9) Techet, A. H. and Triantafyllou, M. S., "Boundary Layer Relaminarization in Swimming Fish", Proceedings of the Ninth (1999) International Offshore and Polar Engineering Conference, Brest, France, May 30 - June 4, 1999.

10) Techet, A. H., Zhang, X., Wolfgang, M. J., Kumph, J. M., Hover, F. S., Yue, D. K. P., Triantafyllou, M. S., Anderson, E. J., McGillis, W. R. and Grosenbaugh, M. A.: "Flow Control of Flexible-Hull Vehicles", Proceedings of the Eleventh International Symposium on Unmanned Untethered Submersible Technology, (1999), pp.162-171. 
11) Barrett, D. S., Triantafyllou, M. S., Yue, D. K. P., Grosenbaugh, M. A. and Wolfgang, M. J.: "Drag Reduction in Fish-Like Locomotion", Journal of Fluid Mechanics, Vol. 392, (1999), pp. $183-212$.

12) Baldwin, B. S., and Lomax H.: "Thin Layer Approximation and Algebraic Model for Separated Turbulence Flows", AIAA $16^{\text {th }}$ Aerospace Sciences Meeting, (1978), pp.1-18, Huntsville, Alabama.

13) Paterson, E. and Stern, F., "Computation of Unsteady Viscous Flow with Application to the MIT Flapping Foil Experiment”, Sixth International Conference on Numerical Ship Hydrodynamics, pp.699-719, 2-5, August 1993, Iowa Memorial Union, Iowa City, Iowa, USA.

14) Chen, Z. and Doi, Y.: "Numerical Investigation on Propulsion by Undulating Plate", Journal of The Society of Naval Architects of Japan, Vol. 188, (2000), pp.359-366.

15) Abbott, IRA H., "Theory of Wing Sections -..Including a Summary of Airfoil Data", Dover Publications, INC. New York, 1959.

16) Gregory, N. and O'Reilly, C. L., "Low Speed Aerodynamics Characteristics of NACA0012 Airfoil Section. Including the Effects of Upper Surface Roughness Simulation Hoarfrost", National Physical Laboratory, Teddington, England, Aero Report 1308, 1970. 